\title{
Artificial Neural Network Analysis of Microwave Spectrometry on Pulp Stock: Determination of Consistency and Conductivity
}

\author{
Eric C. Green, Student Member, IEEE, Buford Randall Jean, Member, IEEE, and R. J. Marks, II, Fellow, IEEE
}

\begin{abstract}
A method for calibrating a microwave sensor is described. The method utilizes an artificial neural network trained to infer the consistency and conductivity of pulp stock slurry from the measured output spectrum of a microwave instrument. The method is both efficient and robust for extracting the multiple parameter information from the microwave signal output.
\end{abstract}

Index Terms-Microwave sensor, microwave spectrometry, neural network, pulp stock.

\section{INTRODUCTION}

W E PRESENT a method whereby the consistency and conductivity of pulp stock (paper pulp slurry) are determined in real time from broadband microwave spectrometry data using multilayered perceptron neural networks. The method is illustrated with an offline instrument used to calibrate inline pulp stock sensors in a paper manufacturing process. There are a number of commercial microwave sensors that have found application in the pulp stock industry, employing a variety of signal extraction methods such as the time of flight [1], the attenuation and phase shift [2], and the microwave spectrometer method being used here [3].

While there are numerous methods for calibrating a microwave sensor, the process can be formidable. A direct approach is one option, but this process works well only for very simple measurement situations in which the process material's electrical properties are known or can be easily determined. By using established modeling techniques, it is possible to determine a microwave circuit's lumped-element equivalent. From this equivalent circuit, a sensor's output can be predicted and compared to the measured output of the sensing device. The device's output is adjusted as required for correction of measurement errors. Such microwave modeling is an accurate but complex, time consuming, and costly solution. In the design of microwave circuits, the modeling problem itself has been addressed by the application of neural networks [4]. Our application circumvents the modeling process and deals with calibrating the instrument against the desired process variable without the need for precise microwave characterization of the material under test.

Manuscript received May 18, 2005; revised August 25, 2006.

E. C. Green is with the University of Texas School of Law, Austin, TX 78705 USA.

B. R. Jean and R. J. Marks, II are with the Department of Electrical Engineering, Baylor University, Waco, TX 76798-7356 USA (e-mail: Randall_Jean@baylor.edu).

Digital Object Identifier 10.1109/TIM.2006.884284
One simple method using this calibration approach involves visual inspection of microwave spectrometry waveforms generated from multiple measurements taken with a microwave sensor [5]. The observer looks for regions where the curves indicate a distinct response corresponding to known changes in the desired output parameter (Fig. 1). Samples of the measured substance are prepared to known values or analyzed according to the accepted industry methods and standards, and these known values are aligned with the values of the sensor's output readings as experimentally measured for each sample. A calibration curve is then generated by performing a simple linear regression of the known or observed parameter values versus the measured properties (e.g., slopes and offsets of bestfit straight lines) of the spectrometry data from only the visually selected region. This procedure requires that a person select the region of the waveform that is most representative of the desired sensor measurement, a judgment call that may yield a less precise calibration. This method discards spectrometry data with nonobvious relationship to the desired measurement, and therefore, valid calibration information about the sample may be lost, causing measurement inaccuracy. Additionally, it is possible that no single region of the data exhibits a clear and distinct response to changes in the desired output parameter. The neural networks presented here incorporate all spectrometry data and therefore do not have these limitations.

A neural network is an adaptive mathematical framework that can determine nonobvious and nonlinear relationships among data [6]. The basic framework of an artificial neural network can be seen in Figs. 2 and 3. This architecture and workings of such neural networks are well known and are described in detail elsewhere [6]. The architecture shown in Fig. 3 feeds an input of 101 inputs into a hidden layer of ten neurons (or nodes), which, in turn, is broadcast to two neurons in a second hidden layer. The outputs of these two nodes are combined into the single output. A "cut-and-try" approach was used to arrive at the structure. While this approach may not be particularly satisfying for the purist, the overall performance of the trained networks provided excellent results for the application. The use of the sigmoidal activation function is typically a default in neural network architectures of the type used and proved to be successful in the characterization of the data. The commercially available PC-based software package, i.e., QwikNet, was used to design and train the neural networks reported here [7]. The software allows specification of as many as five hidden layers, with each having any number of neurons. 


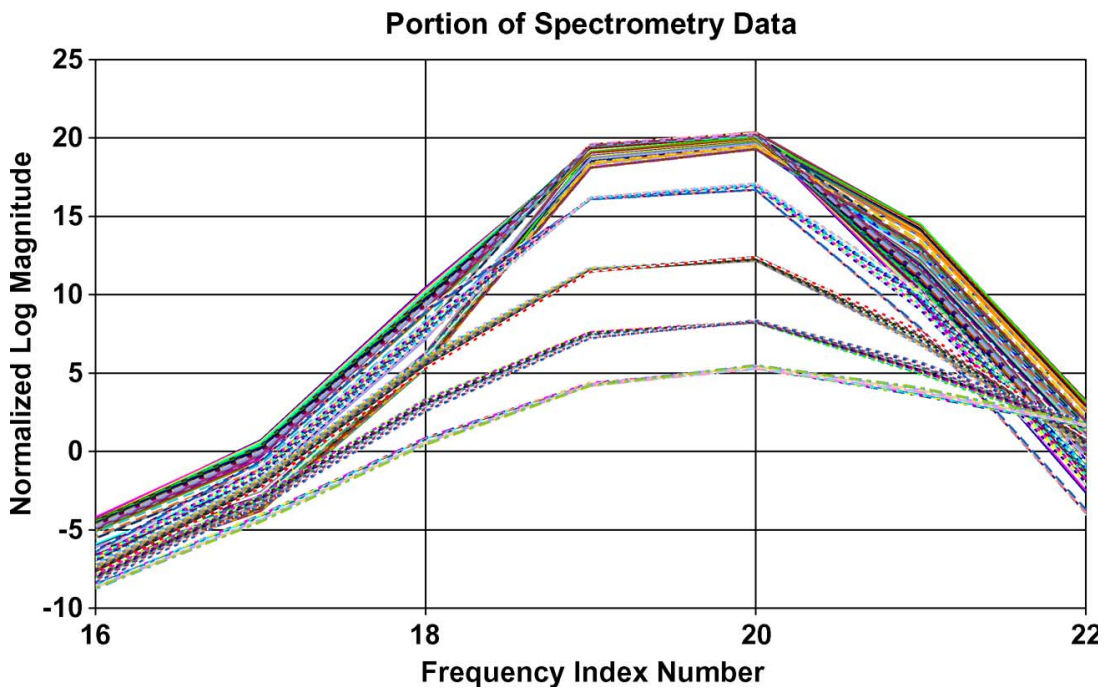

Fig. 1. Visually selected region of 184 microwave spectrometry waveforms used to calibrate an instrument. (Color version available online at http:// ieeexplore.ieee.org).

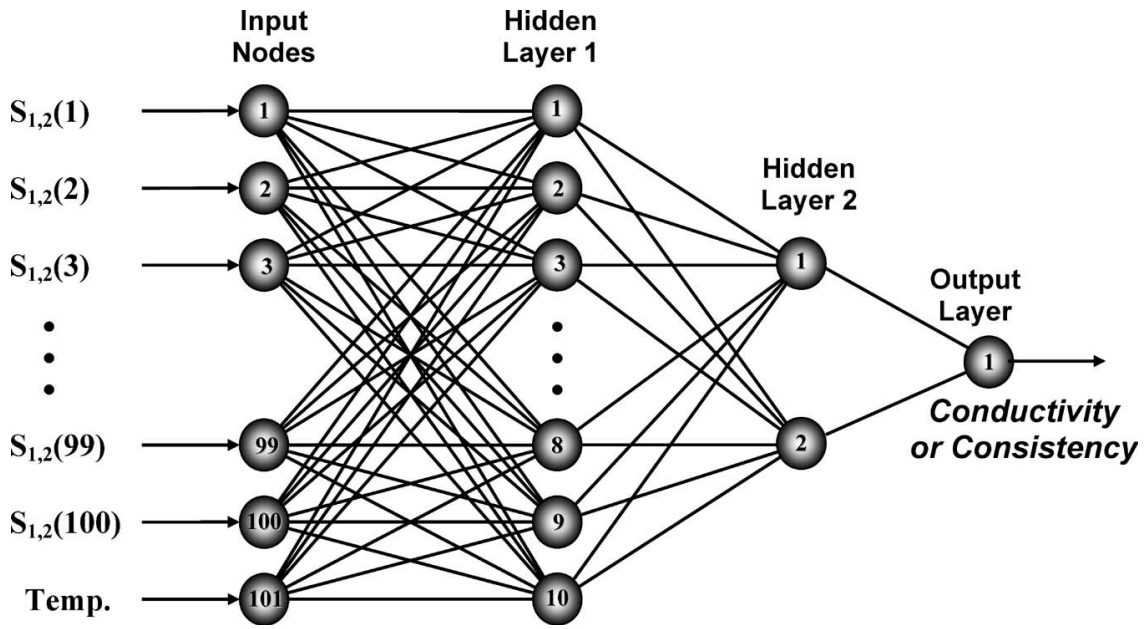

Fig. 2. Artificial neural network setup.

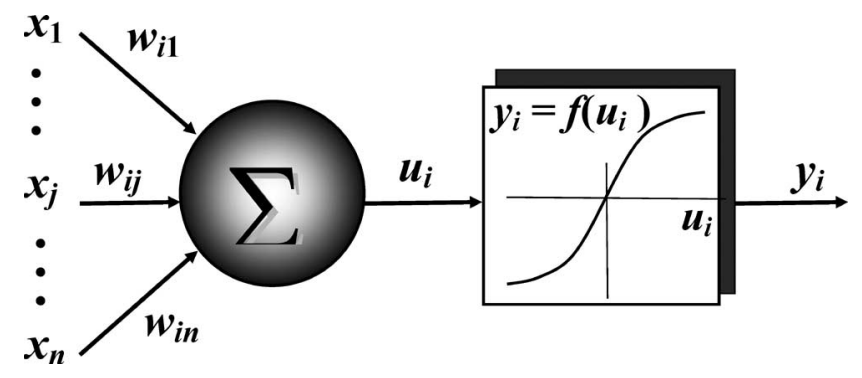

Fig. 3. Typical node. The nonlinear activation function is $y_{i}=f\left(u_{i}\right)$.

The activation function can also be selected from several alternatives including sigmoid (logistic), tanh, Gaussian, and linear.

Neural networks are being used increasingly in the field of microwave engineering. Researchers at the National Institute of Standards and Technology have used a neural network to obtain a full open-short-load-through calibration for a vector network analyzer based on only a few sampled frequency points [8]. One group utilized neural networks in measuring the dielectric constant of a composite dielectric liquid at a specific frequency based on capacitive measurement of the sample [9]. Neural networks have also been implemented to quickly perform difficult inversions of Maxwell's equations so that complex dielectric characterization of a material can be obtained in real time [10], [11].

The type of sensor used in our application has been found to significantly benefit from neural network processing of the raw output data.

\section{Materials And Methods}

Data used to illustrate neural network microwave sensor calibration are from prepared samples of pulp stock typical of that used in paper production. The medium in which the pulp stock is suspended can be water or a filtrate; thus, samples of both were used. Sample preparation involved weighing strips of dried pulp having known antecedent moisture content, adding the strips to a known weight of water or solute, and blending the mixture to separate the pulp fibers and distribute them uniformly within the liquid. The samples were subsequently agitated by a small stirring propeller inside the sample chamber 


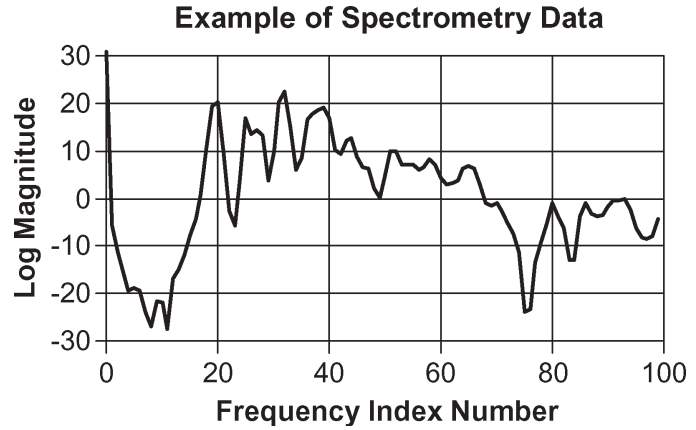

Fig. 4. Microwave spectrometry data for consistency of $0.648 \%$ and conductivity of $200 \mu \mathrm{S} / \mathrm{cm}$.

immediately prior to the signal collection operation to help offset any settling effects that may have occurred. The samples were also pressurized to about $2 \mathrm{~kg} / \mathrm{cm}$ to force any air bubbles in the pulp samples into suspension. Multiple readings were averaged in both time and spectral domains to reduce errors due to any nonhomogeneous distribution of the pulp or due to electronic noise.

The sample chamber was essentially a section of circular waveguide approximately $7.62 \mathrm{~cm}$ in diameter $\times 30.38 \mathrm{~cm}$ in length. The microwave signal was communicated to the pulp sample by means of coupling loop antennas arranged on one side of the waveguide, mounted in ceramic dielectric seals, and spaced about $10 \mathrm{~cm}$ apart. The data consist of samples of the log magnitude transmission spectrum from the microwave sensor at 100 equally spaced frequencies from dc to about 1.2 GHz. The temperature of each sample was also recorded. The pulp stock samples were carefully prepared in the laboratory to assure precise control of their conductivity and consistency and to span the range of conditions that the sensor will encounter for an offline calibration application. Consistency values ranged from $0 \%$ to $1.5 \%$. Consistency is defined as the weight of the dry fiber divided by the total weight of the fiber and solute, expressed as a percentage. The conductivity values covered the span from about 0 to $2000 \mu \mathrm{S} / \mathrm{cm}$. A representative example of the spectrometry data for a consistency of $0.648 \%$ and a conductivity of $200 \mu \mathrm{S} / \mathrm{cm}$ at $21.4{ }^{\circ} \mathrm{C}$ can be seen in Fig. 4.

After normalizing the data discussed above, two neural networks are trained with temperature and spectrometry data inputs and conductivity and consistency outputs. The networks are identical in structure, consisting of 101 input nodes, two hidden layers of ten and two nodes, respectively, and one output node (Fig. 2). The activation function of the hidden layers is logistic, and the activation function of the output layer is linear. The conductivity and consistency networks were trained using the online backpropagation algorithm [6]. Two independent networks were used rather than a single network having two outputs, simply to make observing the dynamics of the training process for each output easier.

\section{RESULTS AND DISCUSSION}

The results of the neural network processing of the data are shown in Figs. 5 and 6. Both of the networks show excellent performance. The performance on conductivity was

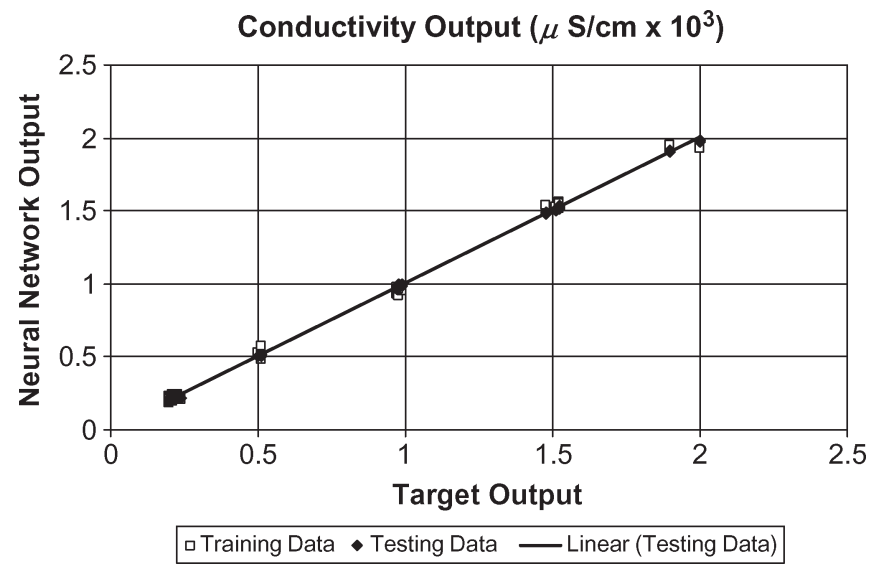

Fig. 5. Graph of the output of the conductivity neural network versus the target data.

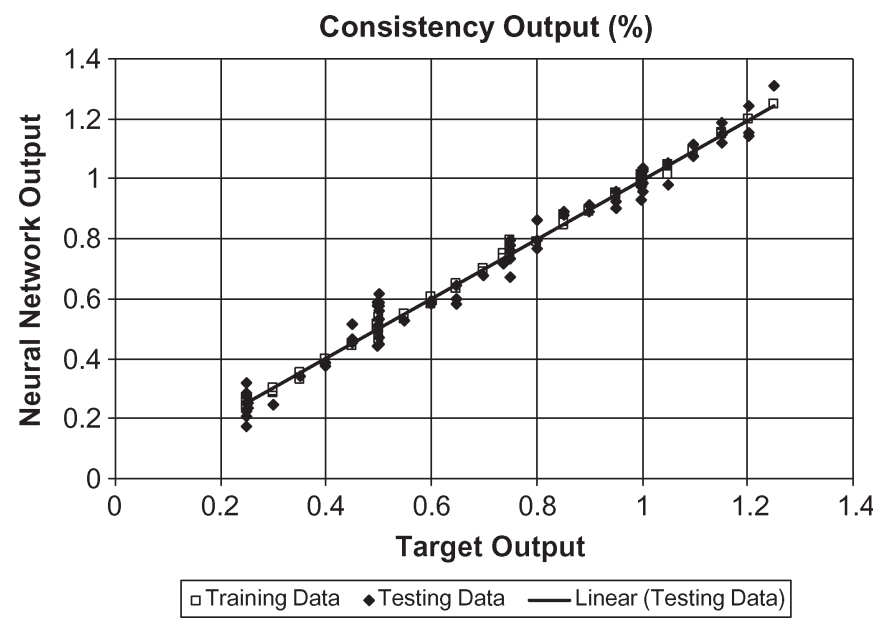

Fig. 6. Graph of the output of the consistency neural network versus the target data.

anticipated since earlier work with these data yielded excellent results with a simple line-fit calibration procedure detailed in [5]. The performance on consistency, however, represented a substantial improvement over the earlier calibration method. Without the benefit of the neural network, the earlier calibration routine required knowing (measuring) conductivity first and then selecting conductivity-specific calibration coefficients for producing the consistency output. This two-step process gave good performance but was labor intensive in its development; in addition, the segmented calibration profile left open the possibility of large errors for intermediate values of conductivity, i.e., for values between the actual calibration points. The neural network calibration of consistency considered all the conductivity values at one time while still producing superior measurement results.

Analysis on the output of test data conductivity neural network showed a correlation coefficient of 0.9983 and a standard error of $25.10 \mu \mathrm{S} / \mathrm{cm}$ over the conductivity span of $1800 \mu \mathrm{S} / \mathrm{cm}$ or $1.25 \%$ full-scale error (Fig. 5). Test data are representative data that are submitted to a trained neural network to determine the response to data the network has not seen before. This process, called cross validation, is a standard testing of the accuracy of a trained neural network's performance [6]. Similarly, 
an analysis on the output of the consistency neural network showed a correlation coefficient of 0.9837 and a standard error of $0.0403 \%$ over the consistency span of $1.00173 \%$ or $3.22 \%$ full-scale error (Fig. 6). As a means for comparison, a multiple linear regression procedure operating on a visually selected region of the waveform, as described above, was performed on the same set of data. The initial correlation coefficient from that procedure was 0.8804 with a standard error of $0.104 \%$. Later attempts with the linear regression method gave somewhat improved results but did not match the performance of the neural network.

\section{CONCLUSION}

The successful application of microwave sensors to a variety of industrial measurement problems often involves dealing with measurement situations that are not well controlled. Extracting the parameter of interest from a given sensor's output often requires considerable experience and tenacity in sifting through large amounts of data. Through its ability to recognize patterns, an artificial neural network is able to automatically sort between needed versus useless data and to obtain a meaningful result without requiring the input of an experienced expert. This characteristic of a neural network makes it particularly useful in the interpretation and analysis of microwave spectrometry data and greatly simplifies the application of this measurement tool.

\section{REFERENCES}

[1] P. Jakkula and E. Tahkola, "Microwave sensor system for the consistency measurement in the pulp and paper industry," in Sensors Update, vol. 7, K. Kupfer, A. Kraszewski, and R. Knøchel, Eds. Hoboken, NJ: WileyVCH, 2000, ch. 8, pp. 211-232.

[2] Y. Shimokawa and M. Suzuki, "Densitometer using microwaves," U.S. Patent 6260 406, Jul. 17, 2001.

[3] B. R. Jean, "Guided microwave spectrometry for in-line analysis of flowable materials," in $R F$ and Microwave Sensing of Moist Materials, Food, and Other Dielectrics, vol. 7, K. Kupfer, A. Kraszewski, and R. Knochel, Eds. Weinheim, Germany: Wiley, 2001.

[4] Q. J. Zhang and K. C. Gupta, Neural Networks for RF and Microwave Design. Norwood, MA: Artech House, 2000.

[5] B. R. Jean, "Process composition monitoring at microwave frequencies: A waveguide cutoff method and calibration procedure," IEEE Trans. Instrum. Meas., vol. 55, no. 1, pp. 180-186, Feb. 2006.

[6] R. D. Reed and R. J. Marks, Neural Smithing. Cambridge, MA: MIT Press, 1999

[7] QwikNet Professional Neural Network Software, Craig Jensen. version 2.23. [Online]. Available: http://qwiknet.home.comcast.net/

[8] J. A. Jargon, K. C. Gupta, and D. C. DeGroot, "Applications of artificial neural networks to RF and microwave measurements," Int. J. RF Microw. Comput.-Aided Eng., vol. 12, no. 1, pp. 3-24, Jan. 2002.

[9] A. Rajendran and P. Neelamegam, "Dielectric constant measurement using an artificial neural network," Instrum. Sci. Technol., vol. 32, no. 4, pp. 413-422, 2004.

[10] E. E. Eves, P. Kopyt, and V. V. Yakovlev, "Determination of complex permittivity with neural networks and FDTD modeling," Microw. Opt. Technol. Lett., vol. 40, no. 3, pp. 183-188, 2004.

[11] R. Olmi, G. Pelosi, C. Riminesi, and M. Tedesco, "A neural network approach to real-time dielectric characterization of materials," Microw. Opt. Technol. Lett., vol. 35, no. 6, pp. 463-465, Dec. 2002.

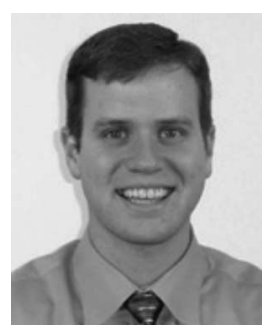

Eric C. Green ( $\left.\mathrm{S}^{\prime} 00\right)$ was born in St Louis, MO, on November 2, 1981, but spent the majority of his life living in Houston, TX. He received the B.S. degree in electrical and computer engineering and the M.S. degree in biomedical engineering from Baylor University, Waco, TX, both in 2006. He is currently working toward the JD degree at the University of Texas Law School, Austin.

$\mathrm{He}$ served as IEEE-USA's Intellectual Property Committee Summer Intern in 2002, as a Legal Assistant Clerk for Baker Botts L.L.P. in 2004, as a Research Assistant to Dr. B. R. Jean from 2004 to 2006, and, most recently, as a Technical Advisor for the law firm Patterson and Sheridan, L.L.P., Houston, where he assisted with patent prosecution. His research while at Baylor involved using neural networks to analyze the output of microwave sensors, and he developed a microwave sensor for noninvasively measuring biometric parameters in humans.

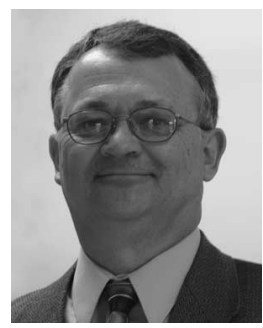

Buford Randall Jean (M'66) was born in Hillsboro, TX, on May 14, 1948. He received the B.S., M.S., and $\mathrm{Ph} . \mathrm{D}$. degrees in electrical engineering from Texas A\&M University, College Station, in 1970, 1971, and 1978, respectively.

For eight years, he was a Faculty Member with the Department of Electrical Engineering, Texas A\&M He left Texas A\&M to form an industrial measurement company in 1986 with several partners. For the next 17 years, he worked to develop, market, and implement several innovative microwave sensing technologies for industrial applications and is the holder of numerous patents in the said field. In 2003, he joined Baylor University, Waco, TX, as an Associate Professor, being attracted to the university by the Baylor 2012 vision. His current research involves both industrial and biomedical applications of microwave and ultrawideband sensors and systems.

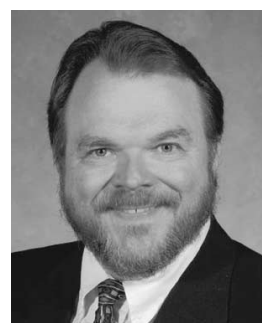

R. J. Marks, II (S'71-M'72-SM'83-F'94) is a Distinguished Professor of electrical and computer engineering with the Department of Engineering, Baylor University, Waco, TX. He has more than 300 publications. Seven of his papers have been reproduced in volumes of collections of outstanding papers. He is the author/coauthor of two books, including Neural Smithing: Supervised Learning in Feedforward Artificial Neural Networks (MIT Press, 1999), and is the editor/coeditor of four other volumes.

Dr. Marks is a Fellow of the Optical Society of America. He served as a Distinguished Lecturer for the IEEE Computational Intelligence Society. He served as the Faculty Advisor with the University of Washington's chapter of Campus Crusade for Christ for 18 years. He served a six-year stint as the Editorin-Chief of the IEEE TRANSACTIONS ON NEURAL NETwORKS. He received the Outstanding Branch Councilor award from the IEEE and was presented with the IEEE Centennial Medal. He was named a Distinguished Young Alumnus of Rose-Hulman Institute of Technology and is an inductee of the Texas Tech Electrical Engineering Academy. In 2000, he was awarded the Golden Jubilee Award by the IEEE Circuits and Systems Society. He is also the first recipient of the IEEE Neural Networks Society Meritorious Service Award and was the first honorary member of the Puget Sound Section of the Optical Society of America. He was also a corecipient of a NASA Tech Brief Award and the Judith Stitt Award from the American Brachytherapy Society 23rd Annual Meeting. 